
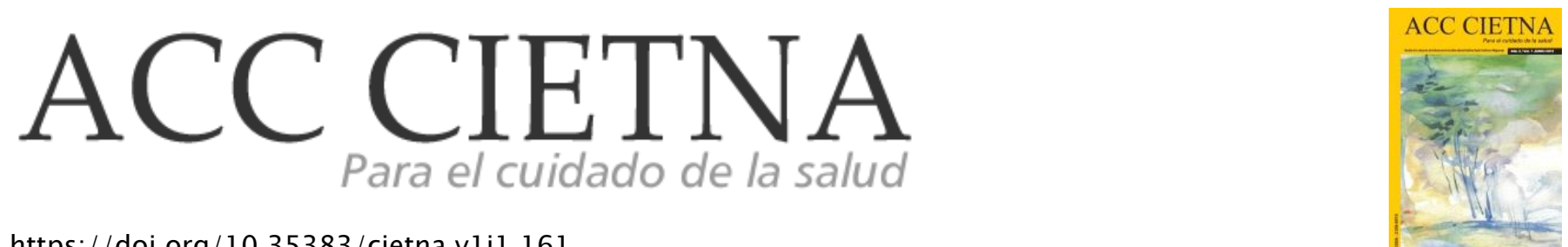

https://doi.org/10.35383/cietna.v1i1.161

\title{
Estilos de cuidar de las estudiantes de enfermería del octavo ciclo en el Hospital Nacional Almanzor Aguinaga Asenjo
}

\author{
Calderón Flores Audrey ${ }^{1}$, Calderón Flores Audrey Falla García Claudia ${ }^{2}$, Vega Ramírez Angélica \\ Soledad $^{3}$
}

\section{INFORMACIÓN DEL ARTÍCULO Historia del artículo: \\ Recibido el 3 de mayo de 2013 \\ Aceptado el 15 de diciembre de 2013}

\section{Palabras claves:}

Estilos

Cuidado

Estudiantes de Enfermería

\section{RESUMEN}

La presente investigación de tipo cualitativo, con abordaje estudio de caso, tiene como objeto de estudio los estilos de cuidar de los estudiantes de enfermería del octavo ciclo en el Hospital Nacional Almanzor Aguinaga Asenjo. Y se realizó con el objetivo de describir, analizar y comprender los estilos de cuidar de tales estudiantes. El sustento teórico se basó en Maya (2001), para estilos y Waldow (2008), para cuidado. Las informantes estuvieron conformados por cuatro enfermeras que laboran en el servicio de Oncología, seis estudiantes que cursaron el octavo ciclo y seis pacientes hospitalizados en el servicio mencionado anteriormente. La muestra se determinó por saturación y se utilizó la entrevista abierta a profundidad según Delgado (1995). En el tratamiento de los resultados se tomó en cuenta el análisis temático según Vítores (2004) emergiendo como gran categoría: estilo de cuidar transformador; y como sub-categorías: estilos de cuidar con pensamiento crítico, estilo de cuidar humanizado, estilo de cuidar empático, estilo de cuidar transcultural involucrando a la familia, estilo de cuidar orientado al desarrollo personal, estilo de cuidar con esmero, estilo de cuidar integral, estilo de cuidar espiritual. Como consideración final se determinó que los estilos de cuidar de las estudiantes de enfermería son transformadores, puesto que tienen en cuenta un cuidado humanizado, que considera a la persona como un ser único e irrepetible, asimismo se le considera un ser en su totalidad y no como la suma de sus partes.

\footnotetext{
${ }^{1}$ Licenciada en enfermería. Clínica Angoamericana- Lima, Perú. Email: acalderon@gmail.com

2 Licenciada en Enfermería. Enfermera con trabajo independiente, Chiclayo, Perú. Email: cfalla@hotmail.com

${ }^{3}$ Magister en Enfermería. Profesora de la Escuela de Enfermería de la Universidad Católica Santo Toribio de Mogrovejo, Chiclayo - Perú. Email: avega@usat.edu.pe
} 
Nursing student care styles for the eighth cycle at the Almanzor Aguinaga National Hospital Asenjo

\section{ABSTRACT}

Keywords:

Styles

Care

Nursing students
The current investigation of kind qualitative, it with case study approach aims to study the styles of care for nursing students in the eighth cycle of the National Hospital Aguinaga Asenjo was aimed to: describe, analyze and understand the types of care for students nursing. The theoretical support fell to Maya(2001) for styles, and Waldow (2008), for caring. The informants were composed of four nurses who work in the Oncology Department, six students who completed the eighth cycle and six hospitalized people, in service mentioned before.The sample was determined by saturation and was used an open interview to depth, according to Delgado (1995). Treatment of the results was taken into account according Vitores thematic analysis (2004) emerging as a big category: transformer and caring style as sub categories: styles of care with critical thinking, caring style humanized care empathetic style, Style transcultural care involving the family, caring style oriented personal development Style take great care, comprehensive care Style, Style spiritual care, taking into account as final consideration styles of care for nursing students are transformers, since they take into account humanized care, considering the person as a unique and unrepeatable, respecting their dignity, coming to treat the person as a whole and not as the sum of its parts.

\section{Introducción}

En la actualidad, los cuidados se orientan a incrementar la interacción positiva de la persona con su entorno, es decir, se concibe el cuidado dirigido al bienestar tal y como la persona lo entiende, entonces la intervención de enfermería con esta orientación va dirigida a dar respuesta a las necesidades de las personas desde su perspectiva holística, respetando sus valores culturales, creencias y convicciones para el éxito de los cuidados, la satisfacción y el bienestar de la persona en su integralidad. A partir de esta opción, los fenómenos que despiertan el interés son las respuestas que presenta el ser humano ante distintas situaciones de salud-enfermedad a lo largo de la vida y esto es, entre otras razones, lo que distingue a la enfermería de otras ciencias ${ }^{1}$.

En consecuencia siempre habrá diferentes formas y/o estilos de cuidar de la enfermera, el cual se perfilará desde la formación del futuro profesional de enfermería, en base a los conocimientos que nuestras teóricas de enfermería nos aportaron. Para las investigadoras el identificar dichos estilos pábulo a realizar este trabajo de investigación, enfocándonos en los estilos de cuidar de las estudiantes de enfermería del octavo ciclo en el Hospital Nacional Almanzor Aguinaga Asenjo, donde las estudiantes realizaron sus prácticas pre 
- profesionales. Zabalza, acota que el período de prácticas pre profesionales es un aspecto importante dentro de la formación académica de los estudiantes, toda vez que en él se inicia el proceso de aproximación a situaciones reales de enseñanza y aprendizaje que tienen varios propósitos, a la vez, sirven de punto de referencia y contraste, para aplicar y revisar conocimientos teóricos ofrecidos desde las diversas disciplinas del plan de estudios. Y esto cobra el mejor de los sentidos si aceptamos como válido que las prácticas se construyen en estrecha interrelación con los contextos educativos, y en conjunción con sus problemáticas, aciertos y desaciertos; además de los condicionantes que ellos suponen para el futuro ejercicio profesional. En este sentido, la práctica "debe ser el hilo rojo", los objetivos sobre los cuales se trabaja constantemente. Premisa que resume gran parte de la insistencia de toda una generación de autores por colocar la práctica pre profesional en el sitial de honor que le corresponde dentro de la formación ${ }^{2}$.

Al intentar reflexionar sobre ello, es necesario analizar cómo es que las estudiantes de enfermería realizan sus prácticas y nos dirigimos específicamente a la manera, forma o estilo de cuidar, los cuales se evidencian durante las prácticas pre-profesionales. Para las investigadoras, actualmente los estudiantes de enfermería que asisten a los servicios sanitarios a realizar sus prácticas, deben ser competentes, en base a conocimiento sólidos, para que de esta manera brinden un cuidado de calidad y con calidez, ya que su relación es más directa con el paciente y pueden prestar ayuda en momentos de tensión, preocupación, gozo o aflicción. Su intervención va dirigida según la disposición propia de la persona cuidada; es por esto que en nuestra realidad, los estudiantes de enfermería para ganar destreza y capacidades; necesitan acudir a un centro hospitalario, siendo esto una forma de aprender, que se pone en práctica desde los primeros ciclos de formación de pregrado en nuestra universidad.
Es en las prácticas hospitalarias donde se pueden evidenciar los estilos de cuidar que adoptan las estudiantes según la influencia del contexto donde se desarrollan y sobre todo orientadas según soportes teóricos y paradigmas acerca del cuidado, y precisamente en estas prácticas donde surge la curiosidad de las estudiantes investigadoras, cuando al realizar las prácticas pre profesionales en las diferentes instituciones de salud, suelen escuchar comentarios como "Muchas veces durante mis horas de práctica, alrededor de la mitad de mi rutina, he culminado con todos los cuidados de enfermería a la persona que se me asignó, sin embargo siento que hay mucho que hacer, pero no sé qué más hacer"; "Solo tengo que esperar cada dos horas para cambiar de posición y nada más"; "Yo no he colocado sondas, quiero hacerlo, porque siento que no sé, y que es importante para mi formación"; "En una ocasión al brindar cuidados a un adulto, él empezó a llorar, pero solo lo escuche, porque no sabía qué hacer, no sé si fue suficiente".

Además se han escuchado comentarios de parte de las personas que reciben el cuidado de los estudiantes, como por ejemplo: "gracias a la señorita estudiante que me ayudó a salir adelante tan solo con su presencia y su sonrisa", "ya llegan las palomas blancas a irradiar alegría y tranquilidad a los pacientes", etc. Frente a la problemática presentada surge en las investigadoras la inquietud por indagar: ¿Cuáles son los estilos de cuidar de los estudiantes de enfermería del octavo ciclo en el Hospital Nacional Almanzor Aguinaga Asenjo?, teniendo como objetivo el describir, analizar y comprender los estilos de cuidar de los estudiantes de enfermería del octavo ciclo.

Considerando que las situaciones cambiantes de la sociedad, de las condiciones de salud y de la dinámica de los servicios obligan a una actualización permanente de competencias, y al mismo tiempo que contribuyen a transformar los servicios. Pero el verdadero poder reside en reforzar el cuidado como eje de la práctica y del 
desarrollo de la disciplina, es así que la investigación encuentra relevancia al existir pocos trabajos de investigación relacionados con los estilos de cuidar de los estudiantes de enfermería y no existe ningún estudio orientado específicamente a profundizar en los estilos de cuidar de los estudiantes en el ámbito hospitalario. Esta investigación impulsó el surgimiento de nuevos aportes sobre esta temática, con la cual se buscó enriquecer el conocimiento y el actuar de los futuros enfermeros frente a la realidad sociopolítica y cultural actual.

La justificación de la presente investigación se basó no solamente en la necesidad de reforzar el cuidado como eje de la práctica y del desarrollo de la disciplina para lograr así una mejor calidad del cuidado; sino sobre todo, en valorar el aporte con el conocimiento específico del cuidado que realiza el estudiante de enfermería pues a veces no es tomado en cuenta, de esta manera procuramos hacer visible lo que es invisible. Se contribuye de esta manera con los soportes teóricos para la enfermera, ya que como se ha mencionado no existen trabajos de investigación cualitativos que se profundicen en el estudio de los estilos de cuidar del estudiante de enfermería en el ámbito hospitalario. Hay que rescatar que las estudiantes del octavo ciclo han adquirido experiencias prácticas en los diferentes servicios, y esto ha permitido describir, analizar y comprender los estilos de cuidar de las futuras profesionales.

Finalmente para las instituciones formadoras de tales estudiantes esta investigación se constituye en un material valioso que permitirá fortalecer $y$ direccionar un proceso formativo encaminado a formar enfermeras que respondan a las necesidades de la sociedad.

\section{Metodología}

Como se ha mencionado es una investigación de tipo cualitativa, en la cual se utilizó la subjetividad de las personas, como sentimientos, emociones, valores, culturas, etc., por lo que permitieron traducir los resultados en apreciaciones conceptuales con mucha precisión o fidelidad posible frente a la realidad estudiada. Esta investigación cualitativa contribuyó a develar el fenómeno de los estilos de cuidado de los estudiantes de enfermería del octavo ciclo en el Hospital Nacional Almanzor Aguinaga Asenjo.

Se utilizó como abordaje metodológico el Estudio de caso, definido por Rodríguez como un examen completo o intenso de una faceta, una cuestión o quizá los acontecimientos que tienen lugar en un marco geográfico a lo largo del tiempo ${ }^{3}$. Por otro lado, Bernal menciona que el objetivo del estudio de caso, es estudiar a profundidad o en detalle una unidad de análisis específica, tomada de un universo poblacional. Para éste método, la unidad de análisis, "el caso" objeto de estudio es comprendido como un sistema integrado que interactúa en un contexto específico con características propias ${ }^{4}$.

En el presente trabajo de investigación, se tomó en cuenta este tipo de abordaje, ya que se pretendió estudiar y registrar los diferentes estilos de cuidar de los estudiantes de enfermería en el lugar donde realizan sus prácticas pre profesionales, tomando como fuentes de información a los estudiantes de enfermería, las enfermeras que observan el cuidado de estas estudiantes y las personas hospitalizadas que lo reciben, situando a los sujetos en un ámbito delimitado como es el contexto de los estudiantes de enfermería en las prácticas pre profesionales, y cuyo objetivo se centró en un caso específico o situacional, porque son un grupo específico, es decir, las estudiantes del octavo ciclo de la Universidad Católica Santo Toribio de Mogrovejo y en una situación específica, es decir durante sus prácticas pre profesionales en el Hospital Nacional Almanzor Aguinaga Asenjo. Así mismo se consideró este abordaje metodológico, ya que la presente investigación contiene un conocimiento por primera vez explorado, siendo este punto de partida para futuras investigaciones. 
Se tuvo en cuenta también los principios del estudio de caso según Ludke5: Los estudios de caso se dirigen al descubrimiento, enfatizan la interpretación en el contexto, buscan retratar la realidad en forma compleja y profunda, usan una variedad de fuentes de información y procuran representar los diferentes y a veces conflictivos puntos de vistas presentes en una situación social.

Las personas entrevistadas fueron un total de 06 estudiantes de enfermería del octavo ciclo de la Universidad Católica Santo Toribio de Mogrovejo. La delimitación se hizo mediante el fenómeno de saturación que consiste en dejar de recolectar información una vez que se repiten los discursos o cuando ya no son significativos para la construcción del objeto de estudio de la investigación. Además constituyeron informantes para develar el fenómeno de los estilos de cuidar de las estudiantes, 06 pacientes hospitalizados en el servicio de Oncología del Hospital Nacional Almanzor Aguinaga Asenjo que recibieron el cuidado y 04 Enfermeras que observaron como la estudiante brindaba el cuidado, permitiendo de esta manera la triangulación de datos en donde se usaron las tres fuentes de información mencionadas anteriormente, logrando obtener diferentes puntos de vista, así como la validación del estudio.

El escenario de la investigación fue el servicio de Oncología del Hospital Nacional Almanzor Aguinaga Asenjo, se procuró en todo momento realizar la entrevista en un ambiente tranquilo libre de ruidos e interrupciones. El instrumento de recolección de datos fue la entrevista abierta a profundidad 6 . El análisis temático se hizo a través de las etapas de Vitores7: Preanálisis, Codificación y Categorización De esta forma se pudo dar significado a todo lo referido durante las entrevistas.

Se tuvo en cuenta en todo momento los criterios de rigor ético según la ética personalista de Sgreccia ${ }^{8}$, los cuales son: Valor fundamental de la vida, principio de Libertad y Responsabilidad y el principio de Sociabilidad y Subsidiaridad. Durante toda la investigación, se siguieron algunos Criterios de Cientificidad, citados en Polit y son la Credibilidad, Confirmabilidad y Transferibilidad o aplicabilidad 9,10 .

\section{Resultados: análisis y discusión}

En la presente investigación de abordaje estudio de caso se describió, analizó y comprendió los estilos de cuidar, a través de categorías y subcategorías, basados en Maya ${ }^{11}$ y Waldow ${ }^{12}$ quienes basan los conceptos en el emic $y$ etic.

El estudio permitió develar como tema central y que constituyó el estilo principal un estilo transformador y subtemas o estilos secundarios: estilo de cuidar con pensamiento crítico, estilos de cuidar humanizado, estilo de cuidar transcultural involucrando a la familia, estilo de cuidar orientado al desarrollo personal, estilo de cuidar con esmero, estilo de cuidar integral y estilo de cuidar espiritual.

Estilo de cuidar transformador, que evidencia los cuidados de las estudiantes de enfermería, el cual busca no dividir según las dimensiones que posee, sino, por el contrario, ver a la persona como un todo y así poder individualizar los cuidados que ella requiera, siempre respetando su dignidad como persona humana y el punto de vista que pueda tener de su salud.

Estilo de cuidar con pensamiento crítico, denota como las estudiantes de enfermería, antes de realizar sus cuidados analizan, para tomar acciones de manera independiente y precisa ante el cuidado a la persona hospitalizada, utilizando elementos que pueda contribuir en el futuro perfeccionamiento de la práctica ${ }^{13}$. El pensamiento crítico es un proceso activo, en donde se hace uso de la mente, para reflexionar y tomar decisiones sobre diferentes situaciones que se pueden presentar, luego tomarse acciones de manera independiente y precisa ante el cuidado a la 
persona hospitalizada, tal como se evidencia en los siguiente discursos:

"En lo que he podido apreciar, las estudiantes realizan sus cuidados siempre con fundamento científico, teniendo en cuenta el ¿Por qué? $Y$ el ¿Para qué? de las cosas" (Enf. Libra).

"Teniendo en cuenta la valoración e historia clínica que utilizo para identificar otra necesidad que está alterada, por ejemplo como la necesidad de higiene, le realizo a la persona un baño completo o de esponja dependiendo de su condición... Al valorar a la persona identifico las necesidades que están afectadas y así dirigir los cuidados que le voy a brindar... La persona que estuvo a mi cuidado, luego de realizarle la valoración completa, le administré los medicamentos en este caso antibióticos, para lo cual aplique los 5 correctos siempre teniendo en cuenta el saludar a la persona y explicarle qué le estoy colocando"(Est. Violeta).

Estilo de cuidar humanizado, las estudiantes de enfermería reflejan en su forma de cuidar, una actitud humana, este cuidado no requiere solo de curar una herida, o aliviar un dolor, pues este busca captar un sentido más, una forma de expresión, de relación de expresión con el otro ser $y$ con el mundo, como una forma de vivir plenamente, con ello se llega a cumplir ayudar y dar cariño, siendo este el más notorio del cuidado $^{14}$. El cuidado es complejo pero a la vez es simple, puesto que con un simple gesto, estamos cuidando, ya que no sólo tenemos que abocarnos a realizar procedimientos técnicos, sino tener un cuidado más cercano con la persona, relacionándonos con el otro ser, tal como se evidencia en el siguiente discurso:

"Es tierno observar con que desinterés y dedicación cuidan las estudiantes, debido a que cualquier persona no cuida de esta manera, ayudando tanto a ella como a su esposo; a ella dándole una muerte tranquila y a él dándole fuerza y resignación... EI consuelo que se le dio a la persona específicamente fue para aliviarle su dolor, sobándole su cabeza, pues no había nada más que hacer para lograr su recuperación... Momentos antes de fallecer la señora, las estudiantes estaban en constante movimiento cuidándola y al momento de fallecer la señora eliminó sangre y las señoritas estudiantes se mancharon su mandil y a pesar de esto ellas no se restringieron ni asustaron ante esto protegieron a la señora $y$ de inmediato limpiaron su mascarilla de oxígeno... Las estudiantes cumplieron su rol, pues ellas cuidaron y auxiliaron a la señora en su último momento de vida... Estoy segura que los cuidados que las señoritas le brindaron a la señora la ayudaron en mucho, porque pienso que ella murió tranquila, por así decirlo, contenta, ya que ellas en todo momento que la cuidaron le hablaban y ella a pesar de su dolor entendía porque movía la cabeza, asi mismo ella sabía y se sentía tranquila porque sentía qué alguien estaba orando por ella" (Pac. Violeta).

Estilo de cuidar empático, expresa una forma de cuidar, con una comprensión a profundidad el mensaje del otro, es decir no solo entender el significado de vivencia de la persona que está recibiendo el cuidado, sino devolver esa comprensión y se sienta realmente comprendido, percibiendo lo que realmente sienten en ese momento, participando en la experiencia de la persona como si fuera propia'1. Esto se evidencia en los siguientes discursos:

"El estudiante de enfermería presta cuidado en cuanto a mis sentimientos, genera confianza $e$ incentiva la fe en Dios, muestra interés en mí persona y hace que mis días sean más llevaderos... El cuidado que brindan es dedicado y se ponen en el lugar de la otra persona, siendo así como unos angelitos que cuidan del paciente" (Pac. Virgo).

"Al brindar los cuidados a la persona, siempre tengo en cuenta la empatía, que significa ponerme en el lugar de la otra persona, por ejemplo lo que está sintiendo en ese momento, o quizás como me gustaría que me traten, de esta manera brindar un mejor cuidado" (Est. Capricornio). 
Estilo de cuidar transcultural involucrando a la familia, las estudiantes de enfermería reflejan la aplicación de la preservación o mantenimiento de los cuidados culturales pero también de negociación o acomodación de los mismos. Para lograr esto último ellos hacen esfuerzo por traspasar las barreras que dificultan el conocimiento mutuo, precisando el entendimiento de sus costumbres y cultura para brindar un cuidado óptimo ${ }^{15}$. Así lo evidencia el siguiente discurso:

"Pregunto por su familia, amigos, que es lo que realizaba cuando estaba bien de salud, por su trabajo u ocupación; para así tener más contacto con la persona y familia y a la vez que me puedan brindar más información... Mi cuidado también se dirigió a comunicarme con su padre sobre las necesidades de su hija y a educarlo para prevenir infecciones mediante el uso de mascarillas...También se buscó que haya una armonía con su padre, que confíe en él y logré que su mamá la visite, logrando así que se sintiera bien durante su hospitalización y que tenga buena relación con sus familiares" (Est. Violeta).

Otro estilo de cuidar que permite el desarrollo de un cuidado Transformador, es el estilo de cuidar orientado al desarrollo personal, los discursos expresan que las estudiantes de enfermería del octavo ciclo, enfoncan sus acciones de cuidado al desarrollo personal, compartiendo ideas con las personas que cuida, permitiendo así que se conozcan tanto el cuidador como la persona cuidada llegando a una interacción mutua, ayudando esto en su autorrealización y/o autocuidado; teniendo una buena interacción individual y grupal, generando en ella diferentes habilidades y destrezas, una buena comunicación y optimas relaciones interpersonales, mejorando se esta manera su potencial, responsabilidad y autoestima $^{16}$. Esto se evidencia en los siguientes discursos:
"El crecimiento y desarrollo tanto de la estudiante como de la persona cuidada, es posible debido a que ambos sujetos comparten ideas, puesto que tu aprendes de ella y ella de ti, en donde las estudiantes enseñan los cuidados que deben tener en casa por ejemplo en cuanto alimentación, mediante consejería, de esta manera prevenir las complicaciones tanto en el hospital como en casa" (Enf. Escorpio).

"Esto contribuye al desarrollo tanto de la estudiante y de la persona cuidada, debido a que ambos aprenden mutuamente, ya que cada manera de manifestar su dolor o sentimientos es diferente en cada persona y eso nos permite saber actuar en cualquier situación y aprende la persona hospitalizada, en base a la educación que se le brinda para mantener su salud" (Est .Amatiel).

Estilo de cuidar con esmero, las estudiantes de enfermería, al brindar un cuidado con esmero se caracterizan, por poner en cumplimiento una obligación o hacer una cosa con sumo cuidado y atención diligentemente con perfección.

Demostrándose esto en los discursos de cada una de las estudiantes, pues las acciones de cuidado que brindan a las personas a su cuidado, Waldow ${ }^{12}$ las califica como una acción de ayuda, el cual consiste en ofrecer cuidados en base a escuchar a los pacientes, tener tiempo para unir la reflexión a la acción, expresar sentimientos, construir y evaluar junto con los pacientes y las familias proyectos de cuidado y curación de acuerdo a sus hábitos y creencias ${ }^{17}$. Esto se evidencia en los siguientes discursos:

"Los días que han venido las estudiantes a la habitación de mi hijo, una de ellas se ha dedicado a su cuidado le ha traído colores, lo ha ayudado a dibujar y ha estado con él en todo momento" (Pac. Stalyn).

"La estudiante de enfermería distribuye su tiempo para poder cuidar a todas las personas por igual, pues cuando nosotros las necesitamos ellas acuden a nuestro llamado" (Pac. Virgo). 
Por otro lado las estudiantes de enfermería realizan actividades dirigidas a cuidar a la persona de una forma integral, basándose en la persona como un ser unitotal, pues no separa sus dimensiones, mucho menos se basa en la enfermedad, siendo esto un aspecto importante para que el cuidado que brindan las estudiantes causen en las personas cuidadas un sentido de confianza y de apoyo, brindándole así cuidados a la persona que lo necesita y a su familiar cuidador indirectamente, dando todo esto a un estilo de cuidar integral ${ }^{11}$.

Así lo evidencian los siguientes discursos:

"El cuidado se inicia con la valoración, identificando las necesidades alteradas, de esta manera priorizar ya sea por necesidades físicas, psicológicas, emocionales, seguido a ello realizar los cuidados y de esta manera satisfacer sus necesidades" (Est. Margarita).

"De acuerdo a nuestra formación veo a la persona de forma holística, con múltiples dimensiones, no nos enfocamos a cuidar solo a su enfermedad". (Est. Capricornio).

Finalmente, las fuentes informantes que participaron en este estudio, reconocen que las estudiantes de enfermería dirigen parte de su cuidado en la dimensión espiritual, planteándose como estilo de cuidar, el espiritual; pues en cada discurso analizado se reconoce que las estudiantes de enfermería, dentro de los múltiples cuidados que realizan, enlazan la parte espiritual, agenciándose de diversas opciones, pero siempre respetando las creencias religiosas de las personas y así hacerlos sentir importantes, queridos y sobre todo amados por un Ser Supremo, el cual nos dio la vida y será nuestro final ${ }^{18}$.

Así lo evidencian los siguientes discursos:

"Al indicar el monitor que la señora había fallecido, las estudiantes comenzaron a orar cogiendo la Biblia, rezaron el rosario y una de ellas le sobaba la cabeza a la señora lo cual indicaba que ellas pedían para que se acerque a Dios en el último momento de su vida" (Pac. Violeta).

"Mis cuidados han permitido que la persona consiga armonía entre su cuerpo, alma y espíritu" (Est. Violeta).

"El niño y su papá muchas veces me pregunta que si va a morir, en ese momento trate de hablarle de Dios, tanto a él como a su familia, tratando de interactuar con ellos, que son las personas que están más cerca al niño, también doy frases, como que se refugien mucho en Dios, que él lo es todo" (Est. Fergie).

\section{Consideraciones finales}

Abordar el tema: Estilo de cuidar de los estudiantes de enfermería del octavo ciclo en el Hospital Nacional Almanzor Aguinaga Asenjo, desde un punto de vista cualitativo, favorece describir, analizar y comprender los estilos o formas de cuidar de los estudiantes de enfermería en sus prácticas pre profesionales.

Los estilos de cuidar de las estudiantes de enfermería en el Hospital Nacional Almanzor Aguinaga Asenjo, se brindan con una postura transformadora, es decir teniendo en cuenta un pensamiento crítico. Antes de brindar su cuidado se organizan, examinan cada situación haciendo uso del análisis para establecer conclusiones, tomar decisiones, efectuar deducciones y reflexionar, teniendo muy presente un cuidado humanizado en todo momento, viendo la integralidad de la persona, poniendo esmero en el cuidado brindado; asimismo se pone cuidado en el entorno, especialmente de su familia involucrando en su cuidado, se respetan sus creencias, costumbres y mitos, buscando una interacción para su desarrollo personal y brindando cuidado en todas sus dimensiones, como la dimensión espiritual, hasta en los últimos minutos de vida de la persona. 
Al brindar su cuidado, las estudiantes de enfermería no solo tienen en cuenta el curar una herida o aliviar un dolor, por el contrario buscan captar un sentido más, una forma de expresión, ayudando y dando cariño a la persona que cuidan, reconociendo a cada persona como única $e$ interesándose por él, valorar sus actitudes, aptitudes, intereses y motivaciones y además los conocimientos que requiere: su manifestación como persona única, auténtica, capaz de generar confianza, serenidad, seguridad y apoyo efectivo.

Con el cuidado brindado por las estudiantes de enfermería, teniendo en cuenta a la persona como única, con múltiples dimensiones, permite reconocer su cuidado, como transformador, es decir; ayuda para cuidarse pues el es el principal participe del cuidado de su salud.

\section{Referencias bibliográficas}

1. Kérouac, S. El pensamiento Enfermero. Madrid: Masson; 2002.

2. Zabalza M. La enseñanza universitaria: EI escenario y sus protagonistas. Madrid: Narcea; 2002.

3. Rodríguez Gómez, G. Metodología de la Investigación Cualitativa. Málaga: ALJIBE; 1996.

4. Bernal T. Metodología de la investigación: para administración, economía, humanidades y ciencias sociales; 2006.

5. Ludke, M. M. Pesquisa en Educacao: Abordagens Cualitativas. $1^{\text {a }}$ Ed. Brasil; 1996.

6. Delgado, M. Métodos y Técnicas Cualitativas de Investigación en Ciencias Sociales. 1ra ed... Madrid; 1995.

7. Vitores, Ana. Curso de Investigación Cualitativa: fundamentos, técnicas y métodos. Homepage. España. antalya: google.com; 2004. Acceso el 12 de mayo del 2009. Disponible en: http://antalya.uab.es/liniguez/aula/ic_analisis_in formacion.pdf.

8. Morse J. Asuntos Críticos en los Métodos de Investigación Cualitativa. Colombia: Universidad de Antioquia; 2003.

9. Bustamante A. Marcelo. Temas de Bioética: Limitación terapéutica y conflictos éticos. Proporcionalidad. Revista Argentina de Medicina Preventiva; 2006.

10. Polit. Investigación Científica en la Ciencias de la Salud. $6^{\text {a }}$ Ed. México: McGraw - Hill Interamericana; 2002.

11. Maya, L. Estilos saludables: componentes de la calidad de vida; 2001.

12. Waldow, R. Cuidar: Expresión Humanizada. Brasil: Ediciones Palabra; 2008.

13. Potter, Patricia A.; Griffin Perry, Anne. Fundamentos de enfermería: teoría y práctica. HarcourtBrace .3 ed. Madrid; 1996.

14. Brusco, A. Humanización de la asistencia al enfermo. Salterrea.1 ra ed. Madrid-España; 1999.

15. MarrinerTomey A; Raile Alligood M. Modelos y Teorias en enfermería. 5ta ed...Madrid: Elsevier España; 2003.

16. Brito Challa. Desarrollo Personal. Hispanoamérica; 2011. Acceso el 14 de setiembre del 2011 . Disponible en: http://www.degerencia.com/ tema/desarrollo_personal.

17. Diccionario de la real academia. España; 2010. Acceso el 03 de septiembre del 2011. Disponible en: http://diccionario.terra.com.pe/cgi-bin/b.pl

18. Hombre y filosofía una mirada desde la mística. Córdova; 2006. Acceso el 06 de septiembre del 2011. Disponible en: http:// 
bdigital.uncu.edu.ar/objetos_digitales/2651/rieg

o.pdf 